\title{
AGGRESSIVE PERIODONTITIS: A REVIEW
}

\author{
Dr. Baljeet Singh ${ }^{1}$ Dr. Avnika Garg ${ }^{2}$ Dr. Rahul K. Garg ${ }^{3}$ \\ ${ }^{1}$ Prof. \& Head Department of Periodontics, Bhojia Dental College and Hospital, Distt. Solan, Himachal Pradesh (India) \\ ${ }^{2}$ PG Student Department of Periodontics, Bhojia Dental College and Hospital, Distt. Solan, Himachal Pradesh (India) \\ ${ }^{3}$ MBBS, MBA (IIM Ahmadabad) (India) \\ Corresponding Author: \\ ${ }^{1}$ Mobile: 919814510711 Email: baljeet065_singh@yahoo.co.in
}

\begin{tabular}{|l|}
\hline Received : \\
$10^{\text {th }}$ June, 2013 \\
Accepted: \\
$27^{\text {th }}$ August, 2013 \\
$\begin{array}{l}\text { Available online: } \\
28^{\text {th }} \text { Dec, } 2013\end{array}$ \\
\hline
\end{tabular}

\section{INTRODUCTION}

Periodontitis is defined as "an inflammatory disease of the supporting tissues of the teeth caused by specific microorganisms or group of specific microorganisms, resulting in progressive destruction of the periodontal ligament and alveolar bone with pocket formation, recession or both. ${ }^{1}$ Aggressive periodontitis may be universally distinguished from chronic periodontitis by the age of onset, the rapid rate of disease progression, the nature and composition of the associated sub gingival micro flora, alterations in the host's immune response, and a familial aggregation of diseased individuals. ${ }^{2}$ In addition, a strong racial influence is observed in the United States; the disease is more prevalent among African Americans. ${ }^{3}$ Aggressive periodontitis describes three of the diseases formerly classified as "early-onset periodontitis (EOP)." EOP can be practically divided into three groups; prepubertal periodontitis, juvenile periodontitis and rapidly progressive periodontitis. ${ }^{4}$ Incidental attachment loss is a term used only in epidemiological studies to describe severe destructive lesions which affect isolated teeth without meeting the criteria for other forms of EOP. This category includes attachment loss adjacent to an impacted third molar. ${ }^{5}$

\section{ABSTRACT}

Aggressive periodontitis, which encompasses a number of clinical entities, probably results from the mobilization of tissue's destructive mechanisms which are common to most forms of periodontal diseases. The unique attributes of the disease process are due to the virulence of the pathogens and the host susceptibility may be due to the

Keywords: Periodontitis, Aggressive Periodontitis, Periodontal diseases.

\section{PRE-PUBERTAL PERIODONTITIS (PPP)}

Severe, rapid periodontal destruction and tooth loss occur infrequently in children and teenagers and can be classified in two groups according to the age of onset: prepubertal forms, which occur before 11 years of age, although they may extend beyond this period, and the pubertal and adolescent forms, which are seen approximately between 11 and 19 years of age. The term juvenile periodontitis is used to refer to the second group. Both prepubertal and pubertal forms have been sub-classified into localized and generalized forms, depending on their distribution in the mouth. ${ }^{6}$ This is an extremely rare form of periodontal disease characterized by rapid periodontal destruction of the primary dentition. ${ }^{4}$ The disease begins about the age of 4 years or before, during or immediately after eruption of the primary teeth, but may affect the permanent dentition. ${ }^{7}$ Primary teeth are lost by the age of 5 or 6 years. The permanent teeth then erupt normally but within few years destructive periodontal disease affects all the teeth except the third molars. By the age of 15 most of the permanent teeth are lost. ${ }^{8}$ An extremely acute inflammation and proliferation of the gingival tissues, with rapid destruction of bone, are found. ${ }^{9}$ In many instances there is a familial pattern to the disease, and most if not all cases are probably genetically mediated. ${ }^{4}$ There is tendency for the disease to be refractory to antibiotic therapy, frequent respiratory infections and destruction of the roots of teeth. 
Normally children do not present with alveolar bone resorption characteristic of periodontitis. They may at the most, have gingivitis especially during puberty. ${ }^{8}$ In PP, there is an abnormality in chemotaxis of either blood neutrophils or monocytes or both, resulting from an inability of these cells to adhere to surfaces. Histological features of generalized PP show an absence of neutrophils from the gingiva, but a dense lymphoid cell infiltrate consisting of plasma cells is present in the connective tissue. Thus, extensive bone loss may occur in the absence of neutrophils. ${ }^{7}$ The flora inhabiting pockets of individual with prepubertal periodontitis has not been studied nor have serum antibody titers been determined. ${ }^{10}$

\section{RAPIDLY PROGRESSIVE PERIODONTITIS (RPP)}

Rapidly progressive periodontitis (RPP) is characterized by severe generalized periodontal destruction, and may affect any or all of the permanent dentition of patients between the ages of 20 and 35 years. On present available evidence it is difficult to justify the classification of this condition as a separate disease entity, as it could well represent a rapidly progressive chronic periodontitis in a susceptible individual. It is also important to distinguish this condition from post-juvenile periodontitis, and to remember that some conditions, such as Down's syndrome, have a high susceptibility to severe rapidly progressive periodontitis in the permanent dentition. ${ }^{4}$

It may be associated with systemic disease and be refractory to all forms of therapy. Most patients with RP demonstrate abnormalities in peripheral blood neutrophil function that are manifested in an impaired chemotactic ability. ${ }^{7}$ Distribution of lesions does not show any definite pattern. It may be localized to few teeth or may be generalized. ${ }^{8}$ The features of RPP include an active phase of severe, rapid bone destruction involving most of the teeth; spontaneous or rapid slow-down of the process; proliferation and severe inflammatory response during active phase; functional defects in neutrophils or monocytes in the majority of affected patients; the possibility of previous juvenile periodontitis; and systemic disturbances that may include weight loss, mental depression and general malaise. Treatment consisting of root planing and antibiotic therapy is generally effective. ${ }^{7}$ Patients with chronic periodontitis may develop rapidly progressive periodontitis in one or more sites. Since RPP does not have any characteristic diagnostic feature, only periodic evaluation of the rate of tissue destruction will help in proper diagnosis. ${ }^{8}$

\section{LOCALIZED AGGRESSIVE PERIODONTITIS (LAP) \\ (Localized Juvenile Periodontitis/ Deep Cementopathia/ Parontitis marginalis progressive/ Familial juvenile periodontitis/Idiopathic Juvenile Periodontitis/ Periodontosis)}

\section{Historical Background}

In 1923, Bernard Gottlieb reported a patient with a fatal case of epidemic influenza and a disease that he called "diffuse atrophy of the alveolar bone." 11 In 1928, he attributed this condition to the inhibition of continuous cementum formation, which he considered essential for maintenance of the PDL fibers. He then termed the disease "deep cementopathia" and hypothesized that this was a "disease of eruption" and that cementum initiated a foreign body response. ${ }^{12}$ In 1938, Wannenmacher described incisor-first molar involvement and called the disease "parodontitis marginalis progressiva." Contrary to others at that time, Wannenmacher considered this disease an inflammatory process. ${ }^{9}$ Several explanations evolved for the etiology and pathogenesis of this type of disease. ${ }^{13}$ In 1940 , Thoma and Goldman used the term "paradontosis" to refer to this disease; the initial abnormality was located in the alveolar bone rather than in the cementum and consisted of vascular resorption and halisteresis rather than "lacunar resorption". In 1947, Goldman described vascular resorption and halisteresis in a spider monkey as being due to a degenerative non-inflammatory disease of the supporting structures. In 1942, Orban and Weinmann introduced the term "periodontosis" and, on the basis of one autopsy case studied in detail, described the development of the disease. Most of the above mentioned studies considered "periodontosis" a degenerative disease caused by unknown systemic factors. Glickman in 1952 believed that the conditions described in these studies didn't represent a different type of periodontal disease, but rather extreme variants of destructive processes common to all periodontal disease. ${ }^{9}$ Other investigators denied the existence of a degenerative type of periodontal disease and attributed the changes observed to trauma from occlusion. Finally, in 1966, the World 
Workshop in Periodontics concluded that the concept of "periodontosis" as a degenerative entity was unsubstantiated and that the term should be eliminated from periodontal nomenclature. ${ }^{14}$ The term "juvenile periodontitis" was introduced by Chaput and colleagues in 1967 and by Butler in 1969. ${ }^{15}$ Baer (1971) described juvenile periodontitis as a welldefined clinical entity different from adult periodontitis in that it appears to start around puberty, seems more common in girls, appears to occur in families, and is rapidly progressive. ${ }^{16}$ In 1989 the World Workshop in Clinical Periodontics categorized this disease as "localized juvenile periodontitis" (LJP), a subset of the broad classification of "early onset periodontitis" (EOP). ${ }^{17}$ Under this classification system, age of onset and distribution of lesions were of primary importance when making a diagnosis of LJP. Most recently, disease with the characteristics of LJP has been renamed localized aggressive periodontitis. ${ }^{1}$ A more recent definition by Genco etal in 1986 describes localized juvenile periodontitis as a disease occurring in otherwise healthy individuals under the age of 30years with destructive periodontitis localized to the first permanent molars and incisors not involving more than two other teeth. ${ }^{18}$

\section{ETIOLOGY}

Newman et al (1976) studied the microbiology of periodontosis lesions and found a unique bacterial flora consisting of gram negative anaerobic rods. The organisms were subsequently indentified as Actinobacillus actinomycetemcomitans, Capnocytophaga species and aerobic motile rods especially Wolinella recta. The gram positive organisms included streptococcus species, actinomyces and peptostreptococcus. Saglie etal (1988) reported that A. actinomycetemcomitans play an important role in the pathogenesis of the disease. The important tissue destructive mechanisms demonstrated in Actinobacillus actinomycetemcomitans are the following (Lindhe and Slots):

1. Leukotoxin: Many strains of Actinobacillus actinomycetemcomitans produce leukotoxin which can destroy human polymorphhonuclear leukocytes and monocytes. This will compromise the patient's ability to phagocytose and eliminate invading bacteria and bacterial products.

2. Chemotaxis inhibition: They produce factors which inhibit polymorphonuclear leukocyte chemotaxis. Thus leukocytes are prevented from reaching the site of infection.

3. Endotoxin: A.actinomycetemcomitans releases endotoxin which can induce Schwartzman reactions, platelet aggregation, complement activation and bone resorption. Endotoxin released by Capnocytophaga and Bacteroids are weakly toxic but may induce bone resorption.

4. Enzymes: A.actinomycetemcomitans, Capnocytophaga and Bacteroids produce proteolytic enzymes, which can degrade periodontal connective tissue.

5. Fibroblast cytotoxicity: They also possess a fibroblast inhibitory factor and interfere with collagen synthesis. Gingival healing may be impaired by its action.

6. Polyclonal B-Lymphocyte activation: These organisms possess potent polyclonal Blymphocyte activators. They induce release of lymphokines that mediate inflammatory reaction and bone resorption. ${ }^{8}$

Although majority of the studies implicated A.actinomycetemcomitans as the causative organism in Juvenile periodontitis, some investigations have failed to demonstrate such a causal relationship and have found other organisms like fusobacterium, spirochetes, eubacterium and black pigmented bacteroids. $^{1}$

Gottlieb (1928) first suggested that the underlying cause of periodontosis was a defect in cementum formation. He found that the cementum on the teeth from JP subjects had extensive areas of hypoplasia in both the exposed and intra-alveolar root surfaces. This suggests that the defect is related to impaired cementum formation rather than the pathology of the pocket. This defect in cementum formation could be hereditary, and could be an important etiological factor. ${ }^{4}$

Some immune defects have been implicated in the pathogenesis of localized juvenile periodontitis. Several investigators showed that patients with localized juvenile periodontitis display functional defects of PMNs and/or monocytes. These defects can impair the chemotactic attraction of the PMN to the site or its ability to phagocytose and digest the micro-organisms. These defects may be induced by the invading bacteria. ${ }^{9}$ 


\section{CLINICAL CHARACTERISTICS}

LAP usually has an age of onset at about puberty. Clinically, it is characterized as having "localized first molar/incisor presentation with interproximal attachment loss on at least two permanent teeth, one of which is a first molar, and involving no more than two teeth other than first molars and incisors". A striking feature of LAP is the lack of clinical inflammation despite the presence of deep periodontal pockets and advanced bone loss. Furthermore, in many cases the amount of plaque on the affected teeth is minimal, which seems inconsistent with the amount of periodontal destruction present. ${ }^{19}$ Although the quantity of plaque may be limited, it often contains elevated levels of $A$. actinomycetemcomitans, and in some patients, Porphyromonas gingivalis. As the name suggests, localized aggressive periodontitis progresses rapidly. Evidence suggests that the rate of bone loss is about three to four times faster than in chronic periodontitis. ${ }^{16}$ Other clinical features of LAP may include: distolabial migration of the maxillary incisors, increasing mobility of the maxillary and mandibular incisors and first molars, sensitivity of denuded root surfaces to thermal and tactile stimuli, and deep, dull, radiating pain during mastication probably caused by irritation of the supporting structures by mobile teeth and impacted food. Periodontal abscesses may form at this stage, and regional lymph node enlargement may occur. ${ }^{20}$ Not all cases of LAP progress to the degree just described. In some patients the progression of attachment loss and bone loss may be self-arresting. ${ }^{16}$ In many cases of LAP, the disease appears to be selflimiting. The attachment loss and bone destruction do not spread to other teeth. This phenomenon of selflimiting of disease activity with advancing age is called as "burn-out"phenomenon. ${ }^{21}$

\section{RADIOGRAPHIC FINDINGS}

Vertical loss of alveolar bone around the first molars and incisors, beginning around puberty in otherwise healthy teenagers, is a classic diagnostic sign of LAP. ${ }^{4}$ Radiographic findings may include an "arcshaped loss of alveolar bone extending from the distal surface of the second premolar to the mesial surface of the second molar". Bone defects are usually wider than usually seen with chronic periodontitis. $^{22}$ Frequently, bilaterally symmetrical patterns of bone loss occurs, called as "mirror image pattern". ${ }^{18}$

\section{GENERAL HEALTH}

There seems to be no relationship with any systemic condition, although a somewhat similar dental picture is found in the rare Papillon-Lefevre syndrome but with a generalized distribution and also involving the deciduous dentition. ${ }^{4}$

\section{PREVALENCE AND DISTRIBUTION}

The prevalence of LAP in geographically diverse adolescent populations is estimated at less than $1 \%{ }^{1}$ Ramfjord (1961) reported prevalence of juvenile periodontitis as high as $22 \%$ in India. Miglani et al (1965) reported $0.1 \%$ prevalence of juvenile periodontitis in a sample population from Madras, while, Rao and Tewani (1968) reported 6.8\% prevalence in a hospital population from Bombay. ${ }^{8}$

In the United States a national survey of adolescents age 14 to 17 reported that $0.53 \%$ had LAP. ${ }^{1}$ Loe and Brown (1991) examined 11,000 adolescents aged 14 to 17 years in USA and observed that the prevalence of juvenile periodontitis among black children was $2.05 \%$ while it was $0.13 \%$ in white subjects. Available data suggests that the highest prevalence of juvenile periodontitis is among black males followed in descending order by black females, white females and white males. It is mostly seen between puberty and 20 years of age. ${ }^{8}$

Several investigators have suggested a genetic transmission and, because of the apparently greater frequency in females, an X-linked dominant inheritance has been suggested. ${ }^{4}$ Others considered it to be transmitted as an X-linked dominant disease. ${ }^{9}$

\section{HISTOPATHOLOGY}

Gingival biopsies from LJP patients show a severe inflammatory infiltrate in the gingival epithelium and connective tissue adjacent to the subgingival microflora. Over $60 \%$ of the round cell infiltrate in the lesion is composed of plasma cells. In chronic periodontitis plasma cel1 infiltration is found to be only half of this. Large numbers of infiltrating organisms have been reported in the gingival tissue. Christersson et al (1984) have shown that Aactinomycetemcomitans can be cultured from gingival biopsies obtained from LJP patients. ${ }^{8}$ 
GENERALIZED AGGRESSIVE PERIODONTITIS

(Generalized Early onset periodontitis/ Generalized juvenile periodontitis)

What is now known as 'generalized aggressive periodontitis' was not clearly described until the later part of the $20^{\text {th }}$ century. However, G.V.Black used the terms 'phagedenic pericementitis' and 'chronic suppurative pericementitis' to describe patients who suffered from a rapid destruction of alveolar bone. ${ }^{1}$

\section{CLINICAL CHARACTERISTICS}

Generalized aggressive periodontitis (GAP) usually affects individuals under age 30, but older patients also may be affected. In contrast to LAP, evidence suggests that individuals affected with GAP produce a poor antibody response to the pathogens present. Clinically, GAP is characterized by "generalized interproximal attachment loss affecting at least three permanent teeth other than first molars and incisors." The destruction appears to occur episodically, with periods of advanced destruction followed by stages of quiescence of variable length (weeks to months or years). As seen in LAP, patients with GAP often have small amounts of bacterial plaque associated with the affected teeth. Quantitatively, the amount of plaque seems inconsistent with the amount of periodontal destruction. ${ }^{19}$ Qualitatively, $P$. gingivalis, $A$. actinomycetemcomitans, and Tannerella forsythia (formerly Bacteroides forsythus) frequently are detected in the plaque that is present. ${ }^{23}$ Some patients with GAP may have systemic manifestations, such as weight loss, mental depression, and general malaise. ${ }^{24}$

The radiographic picture in generalized aggressive periodontitis can range from severe bone loss associated with the minimal number of teeth, as described previously, to advanced bone loss affecting the majority of teeth in the dentition. In a study of untreated periodontal disease conducted in Sri Lanka by Loe etal $8 \%$ of the population had rapid progression of periodontal disease, characterized by a yearly loss of attachment of 0.1 to $1.0 \mathrm{~mm}$. A U.S. national survey of adolescents age 14 to 17 reported that $0.13 \%$ had GAP. In addition, blacks were at much higher risk than whites for all forms of aggressive periodontitis, and male teenagers were more likely to have GAP than female adolescents. ${ }^{1}$

\section{POST-JUVENILE PERIODONTITIS}

Post-juvenile periodontitis is seen in patients over 20 years of age, and shows some of the features of localized JP. The main differences are that the gingival condition and bacterial flora resemble chronic periodontitis. The gingival tissues show clinically obvious inflammation associated with supra- and subgingival plaque and calculus. It would seem that the marked attachment loss seen in this condition mainly results from the active phase of JP. It seems likely that when JP comes under control by the development of effective immunity the pockets become colonized by the usual indigenous bacteria associated with chronic periodontitis, which produce clinically obvious inflammation. Further progression of the condition then becomes related to the same factors which operate in chronic periodontitis.

\section{DIAGNOSIS OF JUVENILE PERIODONTITIS}

The early diagnosis of JP depends on careful and regular periodontal examinations of children and young adolescents from the time of eruption of the permanent teeth. This needs to include careful periodontal probing around the first molars and incisors. Full-mouth radiographs may be necessary for comprehensive diagnosis. As the condition has a familial tendency, siblings and offspring of JP patients should always be carefully examined. ${ }^{4}$

In a recent report, Appelbaum and co-workers examined the collagenase activity in the gingival crevicular fluid of 9 IJP (periodontosis) patients, 10 control patients, and 10 individuals with periodontitis. The results indicate that some IJP (periodontosis) patients have significantly more crevicular fluid collagenase than patients in other groups. Whether this relates to the microbiota or to the inflammatory cells is not known.

\section{TREATMENT AND MANAGEMENT}

Aggressive periodontitis, by definition, causes rapid destruction of the periodontal attachment apparatus and the supporting alveolar bone. The responsiveness of aggressive periodontitis to conventional periodontal treatment is unpredictable, and the overall prognosis for these patients is poorer than for patients with chronic periodontitis. ${ }^{25}$

\section{THERAPEUTIC MODALITIES}

Early detection is critically important in the treatment of aggressive periodontitis (generalized or localized) because preventing further destruction is often more 
predictable than attempting to regenerate lost supporting tissues. ${ }^{26}$ One of the most important aspects of treatment success is to educate the patient about the disease, including the causes and the risk factors for disease, and to stress the importance of the patient's role in the success of treatment. ${ }^{25}$

\section{CONVENTIONAL PERIODONTAL THERAPY}

Conventional periodontal therapy for aggressive periodontitis consists of patient education, oral hygiene improvement, scaling and root planing, and regular (frequent) recall maintenance. It may or may not include periodontal flap surgery. ${ }^{27}$

\section{SURGICAL THERAPY}

Resective periodontal surgery can be effective to reduce or eliminate pocket depth in patients with aggressive periodontitis. ${ }^{26}$ Intrabony defects, particularly vertical defects with multiple osseous walls, are often amenable to regeneration with these techniques. Recent advances in regenerative therapy have advocated the use of an enamel matrix protein (Emdogain) to aid in the regeneration of cementum and new attachment in periodontal defects. ${ }^{25}$

\section{ANTIMICROBIAL THERAPY}

The use of systemic antibiotics was thought to be necessary to eliminate pathogenic bacteria (especially A. actinomycetemcomitans) from the tissues. Indeed, several authors have reported success in the treatment of aggressive periodontitis using antibiotics as adjuncts to standard therapy. ${ }^{26}$ The use of local delivery to administer antibiotics also offers a novel approach to the management of periodontal "localized" infections. Local delivery agents have been formulated in many different forms, including solutions, gels, fibers, and chips.

\section{HOST MODULATION}

Variations in host response between individuals are greatly responsible for observed differences in disease severity. This is especially true for individuals with aggressive periodontitis. Several agents have been used or evaluated to modify the host response to disease. The use of subantimicrobial-dose doxycycline (SDD) may help to prevent the destruction of the periodontal attachment by controlling the activation of matrix metalloproteinases, primarily collagenase and gelatinase, from both infiltrating cells and resident cells of the periodontium, primarily the neutrophils.
Other agents, such as flurbiprofen, indomethacin, and naproxen, may reduce inflammatory mediator production. Further research needs to be done to substantiate the effects of these agents. ${ }^{25}$

\section{TREATMENT PLANNING AND RESTORATIVE CONSIDERATIONS}

Successful management of patients with aggressive periodontitis must include tooth replacement as part of the treatment plan. In the patient with aggressive periodontitis, the approach to restorative treatment should be made based on a single premise: extract severely compromised teeth early, and plan treatment to accommodate future tooth loss. ${ }^{1}$

\section{USE OF DENTAL IMPLANTS}

Initially, the use of dental implants was suggested and implemented with much caution in patients with aggressive periodontitis because of an unfounded fear of bone loss. However, evidence to the contrary appears to support the use of dental implants in patients with aggressive periodontal disease. ${ }^{26}$ Currently, the use of dental implants must be considered in the overall treatment plan for patients with aggressive periodontitis. ${ }^{25}$

\section{PERIODONTAL MAINTENANCE}

When patients with aggressive periodontitis are transferred to maintenance care, their periodontal condition must be stable (i.e., no clinical signs of disease and no periodontal pathogens). Each maintenance visit should consist of a medical history review, an inquiry about any recent periodontal problems, a comprehensive periodontal and oral examination, thorough root debridement, and prophylaxis, followed by a review of oral hygiene instructions. If oral hygiene is poor, patients may benefit most from a review of oral hygiene instructions and visualization of plaque in their own mouth before debridement and prophylaxis. ${ }^{26}$

\section{PROGNOSIS}

The prognosis for patients with aggressive periodontitis depends on (1) whether the disease is generalized or localized, (2) the degree of destruction present at the time of diagnosis, and (3) the ability to control future progression. Overall, patients with generalized aggressive periodontitis tend to have a poorer prognosis because they typically have more teeth affected by the disease and because the disease is less likely to go spontaneously into remission 
compared with patients with localized forms of aggressive periodontitis. ${ }^{1}$ Some cases of periodontosis seem to go inexorably toward the loss of all the teeth. Fortunately the number of these hopeless cases is small by our present knowledge. There is an element of uncertainty as far as the prognosis for the health of the remaining teeth is concerned. If dentists and patients are aware of this, many difficulties can be avoided. ${ }^{25}$

\section{REFERENCES}

1. Newman MG, Takei HH, Klokkevold PR and Carranza FA. Carranza's Clinical Periodontology. $10^{\text {th }}$ edition. St. Louis: Saunders; 2006.

2. Hart TC. Genetic risk factors for early-onset periodontitis. J Periodontol 1996;67:355-366.

3. Loe H, Brown LJ. Early-onset periodontitis in the United States of America. J Periodontol 1991;62:608-616.

4. Page R.C., Baab D.A. Anew look at the etiology and pathogenesis of early onset periodontitis. J Periodontol 56:748-751.

5. Jenkins WMM and Allan CJ. Periodontics, A Synopsis. Oxford: Wright; 1999.

6. Watanabe K. Prepubertal periodontitis: A review of diagnostic criteria, pathogenesis and differential diagnosis. J Periodont Res 1990;25:31.

7. Page R.C. et al. Characteristics of early-onset periodontitis and identification of the molecular abnormality in one form. $7^{\text {th }}$ International Conference on Periodontal Research, 1986 Sept;8-11.

8. Varma BRR and Nayak RP. Current Concepts in Periodontics. $1^{\text {st }}$ edition. Delhi: Arya Publishing House; 2000.

9. Page R.C. et al. Prepubertal periodontitis: Definition of a clinical disease entity. J Periodontal 1983;54:257.

10. Rao GR. Textbook of Periodontology. $2^{\text {nd }}$ edition. Chennai: All India Publishers and Distributors; 2001.

11. Gottlieb B. Die diffuse atrophy des Alveolarknochens. Z Stomatol 1923;21:195.
12. Gottlieb B. The formation of the pocket: diffuse atrophy of alveolar bone. J Am Dent Assoc 1928;15:462.

13. Wannenmacher E. Ursachen auf dem Gebiet der Paradentopathien. Zbi Gesant Zahn Mund Kieferheilk 1938;3:81.

14. Ramfjord SP, Ash MM, Ker DA. Editors: World Workshop in Periodontics, Ann Arbor, 1966, University of Michigan.

15. Butler JH. A familial pattern of juvenile periodontitis (periodontosis). J Periodontol 1969;40:115-118.

16. Baer PN. The case for periodontosis as a clinical entity. $\mathbf{J}$ Periodontol 1971;42:516-520.

17. Caton J. Consensus report: periodontal diagnosis and diagnostic aids. In Proceedings of the World Workshop in Clinical Periodontics, Chicago, 1989, American Academy of Periodontology.

18. Tonnetti MS, Mombelli A. Early onset periodontitis. Ann Periodontol 1999;4:39.

19. Lang N, Bartold PM, Cullinan M et al. Consensus report: aggressive periodontitis. Ann Periodontol 1999;4:53.

20. Manson JD, Lehner T. Clinical features of juvenile periodontitis (periodontosis). J Periodontol 1974;45:636640.

21. Thoma KH, Goldman HM. Wandering and elongation of the teeth and pocket formation in paradontosis. J Am Dent Assoc 1940;27:335.

22. Miller SC. Precocious advanced alveolar atrophy. J Periodontol 1948;19:146.

23. Tonetti MS, Mombelli A. Early onset periodontitis. Ann Periodontol 1999;4:39.

24. Page RC, Altman LC, Ebersole JL et al. Rapidly progressive periodontitis: a distinct clinical condition. J Periodontol 1983;54:197-209.

25. Grant DA, Stern IB and Everett FG. Periodontics. $5^{\text {th }}$ edition. Toronto: St. Louis; 1979.

26. Newman MG, Takei HH, Klokkevold PR and Carranza FA. Carranza's Clinical Periodontology. $11^{\text {th }}$ edition. St. Louis: Saunders; 2011.

27. Tanner AC, Socransky SS, Goodson JM. Microbiota of periodontal pockets losing crestal alveolar bone. J Periodontal Res 1984;19:279-291. 